\title{
Aelurostrongylus pottoi n. sp. Métastrongylide parasite de Primates. Remarques sur les affinités entre les Nématodes de Carnivores, d'Insectivores et de Lémuriens
}

\author{
Par Alain-G. CHABAUD et Odile BAIN
}

Un spécimen de Perodicticus potto Müller, provenant de la station de La Maboké (République centrafricaine), a été conservé en captivité à la Ménagerie du Jardin des Plantes du 16 novembre 1963 jusqu’à sa mort, le 12 février 1965. Le Docteur Guy Chauvier, ayant posé le diagnostic de pseudo-tuberculose, remarqua à l'autopsie de nombreuses capsules parasitaires dans tous les viscères, et en particulier le foie. Il nous communiqua aimablement le matériel pour chercher à savoir si les parasites pouvaient être rendus responsables de la forme généralisée qu'avait revêtue l'affection.

Les capsules disséminées dans les viscères sont de grande taille (environ $2 \mathrm{~mm}$ de diamètre). Elles sont constituées souvent par plusieurs loges fibreuses concentriques entre lesquelles se trouve du pus et la partie centrale est généralement occupée par une larve vivante de Nématode. Ces larves, présentant près de la pointe caudale la légère encoche habituelle aux larves de Métastrongylides, nous avons été amenés à faire un examen soigneux des poumons. Nous y avons trouvé effectivement quelques Métastrongylides et avons pu extraire du parenchyme une femelle et deux mâles entiers, ainsi que quelques fragments d'autres spécimens.

\section{Deseription}

Corps très grêle, recouvert d'une gaine tégumentaire résistante, largement décollée de la cuticule. La tête, très étroite $(20 \mu)$, n'a pas été étudiée en vue apicale. Elle est constituée de six (? ou huit) lobes bien saillants. EEsophage très court, cylindrique, un peu enflé à sa partie moyenne et à son extrémité postérieure. Pore excréteur s'ouvrant au niveau de la partie moyenne de l'œsophage chez la femelle, un peu en arrière de l'œsophage chez le mâle. Les deux glandes excrétrices atteignent une longueur de près d'un millimètre (fig. B). 


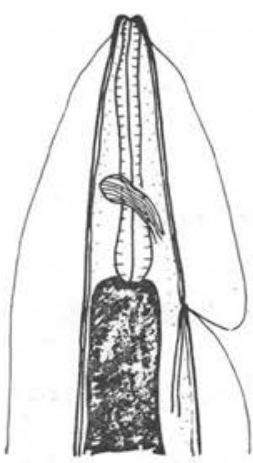

A
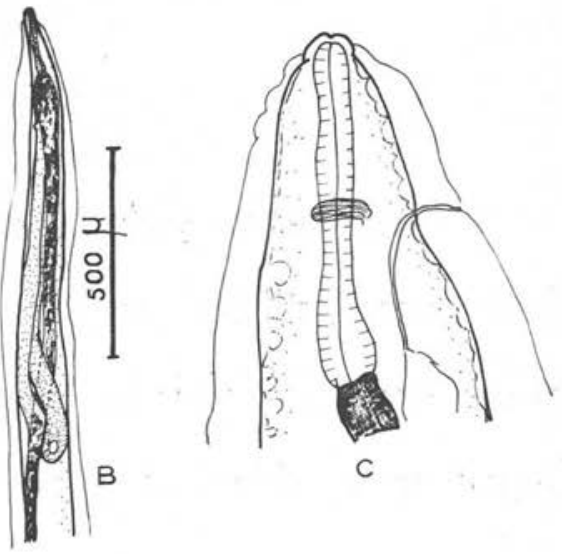

C

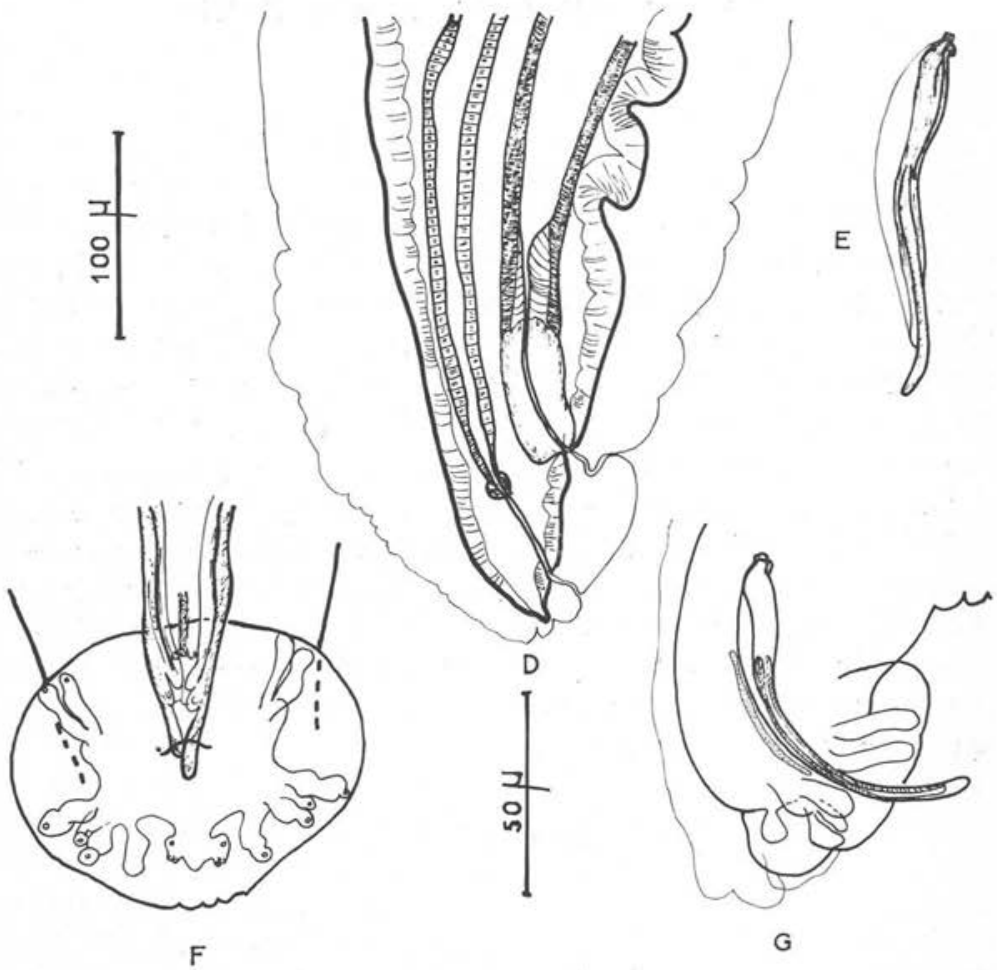

Aelurostrongylus pottoi n. sp.: A) Extrémité antérieure du mâle, vue latérale. - B) Glandes excrétrices du mâle, vue latérale. - C) Extrémité antérieure de la femelle, vue latérale. D) Extrémité postérieure de la femelle, vue latérale. - E) Spicule gauche. - F) Bourse caudale, vue ventrale. - G) Id., vue latérale. (A, C, D : échelle $100 \mu$; B : échelle $500 \mu ; \mathrm{E}, \mathrm{F}, \mathrm{G}$ : échelle $50 \mu$ ) 
Male.

Corps long de 15,5 mm, large de $130 \mu$. Esophage long de $160 \mu$. Anneau nerveux et pore excréteur respectivement à $115 \mu$ et $175 \mu$ de l'apex. Bourse caudale figurée en $F$ et $G$ haute de $65 \mu$ et large de $85 \mu$. Spicules très arqués, longs de $100 \mu$, avec des ailes enflées se terminant à $12 \mu$ de l'extrémité distale arrondie. Gubernaculum long de $35 \mu$.

Femelle.

Corps long de $35 \mathrm{~mm}$, large de $180 \mu$. Esophage long de $170 \mu$. Anneau nerveux et pore excréteur respectivement à $90 \mu$ et $100 \mu$ de l'apex. Extrémité postérieure avec faible mucron arrondi terminal. Anus à $65 \mu$ de la pointe caudale et vulve à $70 \mu$ en avant de l'anus. Provagin absent. L'ovéjecteur impair est long de $450 \mu$; le vestibule, long de $70 \mu$, semble revêtu de cuticule. Il n'y a pas de larves ou d'œufs mûrs chez le spécimen étudié.

\section{Discussion}

Les spicules épais, ailés et fortement courbés, permettent de limiter la discussion aux deux espèces les plus proches: A. falciformis (Schlegel 1933), parasite de Meles en Europe, et $A$. pridhami Anderson 1962, parasite de Mustela au Canada.

A. falciformis peut être reconnu par la forme bifurquée de la côte dorsale et par l'épaisseur du gubernaculum; $A$. pridhami par les côtes ventrales longuement soudées à la base et la taille plus réduite des spicules.

Nous pensons donc que l'espèce du Lémurien est nouvelle et proposons pour elle le nom d'Aelurostrongylus pottoi n. sp.

\section{Remarques sur les affinités entre les Nématodes de Carnivores, d'Insectivores et de Primates}

Deux espèces de Nématodes ont été récoltées chez ce spécimen de Perodicticus potto. L'une, étudiée par Quentin (1965), appartient au genre Molineus, qui, en-dehors de quelques phénomènes de capture chez les Rongeurs, est un genre caractéristique de Carnivores, se retrouvant chez les Insectivores archaïques (Tenrecidae de Madagascar et des Antilles) et chez les Primates primitifs (Lémuriens et Platyrhiniens). L'autre, étudiée ci-dessus, appartient au genre Aelurostrongylus, qui a exactement le même spectre d'hôtes : Carnivores variés, Limnogale (Tenrecidae malgache) et Perodicticus (Lémurien).

Cette affinité étroite entre les Nématodes de Carnivores et d'Insectivores, affinité qui s'étend parfois aux Nématodes de Primates, a déjà attiré l'attention de plusieurs auteurs, car les paléontologistes admettent que les ancêtres des Carnivores (Proto-creodonta) sont particulièrement éloignés des ancêtres Insectivores-Primates (Proto-insectivora). 
Pour Osche (1957), tous les Nématodes hétéroxènes connus chez les Insectivores sont des parasites allochtones, provenant tantôt d'Oiseaux Rapaces, tantôt de Carnivores. Il suppose que l'Insectivore, jouant d'abord le rôle d'hôte de transport entre l'Arthropode, hôte intermédiaire, et le Rapace ou le Carnivore, hôte définitif, est devenu au cours des siècles capable d'assurer la maturation des larves, et s'est donc finalement transformé en hôte définitif.

$\mathrm{Si}$ cette hypothèse rend bien compte d'une partie du phénomène et surtout de l'exemple princeps (Stammerinema soricis Tiner, parasite d'Insectivore, est un Acuaride typique, donc normalement caractéristique d'Oiseau), elle n'explique pas pourquoi nous trouvons une ressemblance remarquable entre Nématodes de Carnivores et Nématodes de Tenrecidae, pour des groupes ayant un cycle évolutif direct comme Uncinaria ou Molineus (Chabaud, Brygoo et Petter, 1965). C'est pourquoi nous présentons aujourd'hui une nouvelle hypothèse.

Nous supposons que les affinités entre parasites de groupes zoologiques très différents peuvent s'expliquer parfois de la façon suivante :

A une période géologique déterminée, les genres parasites actuels, étant déjà en place et ayant une spécificité écologique large, ont pu s'adapter aux seuls Mammifères qui vivaient à cette époque. Par la suite ces parasites ont perdu leur plasticité et ont acquis une spécificité plus stricte ne leur permettant plus de s'adapter aux Mammifères qui sont apparus ultérieurement. Dans le cas qui nous occupe, les tableaux dressés par les paléontologistes (cf. par exemple Piveteau in Grassé 1955) montrent qu'au début du paléocène les trois phylums actuels qui étaient déjà apparus sont les Marsupiaux, les Dermoptères et les Insectivores. L'apparition des «Carnivores » n'est signalée qu'ultérieurement, mais en fait, et contrairement aux autres phylums, la lignée CréodontesCarnivores est parfaitement continue. Dans des publications moins spécialisées, les auteurs préfèrent négliger la distinction Eucréodontes-Carnivores et font naître les Carnivores également au paléocène ( $\mathrm{F}$. Petter, 1963).

Parmi les quatre groupes qui existaient au paléocène, il est logique que les Marsupiaux australiens n'aient pas les parasites caractéristiques des Carnivores ; mais il serait intéressant de rechercher ces parasites chez les Marsupiaux américains et les Dermoptères dont la faune parasitaire reste mal connue. Les premiers Primates étant apparus également très précocement, il est normal de retrouver chez eux certains genres caractéristiques tels que Molineus et Aelurostrongylus.

C'est donc, en conclusion, par l'ancienneté d'apparition des hôtes que nous proposons d'expliquer les analogies remarquables entre les Nématodes parasites, d'une part des Carnivores, d'autre part d'Insectivores primitifs et de Primates primitifs.

\section{Bibliographie}

ANDERSON (R. C.), 1962. - The systematics and transmission of new and previously described Metastrongyles (Nematoda: Metastrongylidae) from Mustela vison. Can. Jl. Zool. 40, 893-920. 
Chabaud (A.-G.), Brygoo (E.-R.) et Petter (A.-J.), 1965. - Nématodes pulmonaires du Limnogale. Ann. Parasit., 40, 467-475.

OSCHE (G.), 1957. - Die «Wirtskreiserweiterung » bei parasitischen Nematoden und die sie bedingenden biologisch-ökologischen Faktoren. Z. Parasitenk., 17, 437-489.

Petter (F.), 1963. - Les Mammifères, 128 p. «Que sais-je ? „, n 1100. Presses Univ. France.

Piveteau (J.) in Grassé (P.-P.), 1955. - Traité de Zoologie, 17 (1), Mammifères, 1.170 p.

Quentin (J.-C.), 1965. - Sur la présence de Nématodes Trichostrongylidae du genre Molineus chez des Rongeurs et chez un Lémurien de la station expérimentale de La Maboké. Bull. Mus., sous-presse.

SCHLegel (M.), 1933. - Die Lungenwurmseuche beim Dachse. Berl. tierärztl. Wochschr., 49, 341-344.

Laboratoire de Zoologie (Vers). Mus. Nat. Hist. Nat. 